\title{
Phototherapy is associated with the decrease in serum globulin levels in neonatal hyperbilirubinemia
}

\author{
JUNWEN ZHENG, CONG WEI, MENG ZHAO and DONGCHI ZHAO \\ Department of Pediatrics and Neonatology, Children's Digital Health and Data Center, \\ Zhongnan Hospital of Wuhan University, Wuhan, Hubei 430071, P.R. China
}

Received July 3, 2018; Accepted October 31, 2018

DOI: $10.3892 /$ br.2018.1166

\begin{abstract}
Previous studies have indicated that phototherapy may be associated with childhood immune disorders in later life. The present study aimed to assess the effects of phototherapy as a risk factor in the decrease in serum globulin (GLB) levels during neonatal hyperbilirubinemia. A total of 430 full-term infants aged between 1 and 28 days, diagnosed with neonatal hyperbilirubinemia, were enrolled in the present study. Neonates with intrauterine infection, genetic abnormalities and congenital diseases were excluded from the cohort. All neonates received single-side phototherapy (halogen lamps for $12 \mathrm{~h}$ per day, for 3 days) and/or intravenous albumin (IVALB; $1 \mathrm{~g} / \mathrm{kg} /$ day, for 2 days) and/or intravenous immunoglobulin (1 g/kg/day, for 2 days). Total serum bilirubin (TSB), albumin (ALB) and GLB levels were examined twice, on the first and fourth days of hospitalization. Neonatal TSB concentrations decreased from $299.6 \pm 83.9$ to $163.6 \pm 57.6 \mu \mathrm{mol} / 1$ following 3 days of intensive treatment $(\mathrm{P}<0.001)$. Pearson correlative analysis indicated that TSB was significantly correlated to the GLB level $(\mathrm{r}=0.249$; $\mathrm{P}<0.01)$, but not with ALB level. There was a significant decrease in GLB levels following phototherapy+IVALB $(\mathrm{P}<0.001)$. The GLB levels decreased to $2-4 \mathrm{~g} / 1$ (10-20\% compared with their baseline levels) and were markedly decreased in infants $>16$ days old compared with those in patients aged $<16$ days $(\mathrm{P}<0.001)$. The decreases in GLB levels observed were $21.3 \pm 4.1$ to $18.5 \pm 4.2 \mathrm{~g} / 1$ in the phototherapy group, and $23.0 \pm 3.9$ to $16.6 \pm 4.5 \mathrm{~g} / 1$ in the phototherapy+IVALB $(\mathrm{P}<0.001)$. The decrease in GLB levels was associated with age (95\% confidence interval; -0.152 , -0.016). The results demonstrated that phototherapy decreased serum GLB levels, particularly in infants aged $>16$ days, while
\end{abstract}

Correspondence to: Dr Dongchi Zhao, Department of Pediatrics and Neonatology, Children's Digital Health and Data Center, Zhongnan Hospital of Wuhan University, 169 Donghu Road, Wuhan, Hubei 430071, P.R. China

E-mail: zhao_wh2004@hotmail.com

Key words: phototherapy, serum globulin, neonates, hyperbilirubinemia, intravenous immunoglobuli additional IVALB treatment promoted the decrease, along with increased age.

\section{Introduction}

Hyperbilirubinemia is a common phenomenon and occurs in $\sim 60 \%$ of full-term newborns (1). Although the majority of cases of neonatal hyperbilirubinemia are physiological, excessive unconjugated bilirubin is a potential neurotoxin and infants should be monitored, to identify those who may develop severe hyperbilirubinemia, and in rare cases, acute bilirubin encephalopathy or kernicterus $(2,3)$.

To prevent the potential toxicity of bilirubin, phototherapy has been suggested for the treatment of neonatal hyperbilirubinemia. In addition, intravenous immunoglobulin (IVIG) and albumin (IVALB) are administrated to neonates with hemolytic hyperbilirubinemia $(4,5)$. IVALB may be effective for preventing the occurrence of complications associated with hyperbilirubinemia, including neuronal developmental abnormalities and blood transfusions (6-9). However, there have been a number of previous studies indicating that neonatal hyperbilirubinemia and/or phototherapy may be associated with infant immune disorders including asthma, thrombocytopenia and arthritis (10-12), or septic shock $(13,14)$. Administration of IVALB serves a key role in preventing neonatal encephalopathy or kernicterus by binding to bilirubin and accelerating its supersession in plasma (7). Conversely, albumin (ALB) peptides may exhibit a cross-linking interaction with the neonatal $\mathrm{Fc}$ receptor $(\mathrm{FcRn})$ and promote $\mathrm{IgG}$ catabolism by saturating FcRn to decrease sustained high serum IgG level (15).

To evaluate the occurrence of humoral immunity injury associated with the administration of phototherapy or IVALB in neonatal hyperbilirubinemia, a retrospective study was conducted, and it was identified that phototherapy and IVALB were risk factors in the decrease of globin (GLB) levels in neonatal hyperbilirubinemia.

\section{Patients and methods}

Patients. The present study was conducted in the neonatal intensive care unit (NICU) in Zhongnan Hospital of Wuhan University (Wuhan, China) between January 2011 and December 2015. A total of 465 full-term infants were 
diagnosed with neonatal hyperbilirubinemia, ages ranging from 1 to 28 days, and 430 cases were enrolled in the study cohort according to modified criteria based on the American Academy of Pediatrics Subcommittee on Hyperbilirubinemia, for the management of hyperbilirubinemia in newborn infants $\geq 37$ weeks gestation age (1): i) Newborns had total serum bilirubin $\geq 100 \mu \mathrm{mol} / 1$ at first day of birth or $\geq 257 \mu \mathrm{mol} / 1$ at 2-28 days following birth; ii) patients exhibited blood group incompatibility (ABO), maternal Rhesus group negative hemolysis or G-6-PD deficiency hemolytic anemia and polycythemia; iii) idiopathic hyperbilirubinemia without cholestasis, and cranial hematoma; iv) confirmed bacterial infection following birth with positive blood culture and increased C-reactive protein. Cases of hyperbilirubinemia lasting $>1$ week due to blood group incompatibility or physiological delay were also included in this study. A total of 25 cases were excluded as they exhibited congenital abnormalities, including subtle dysmorphism of unknown significance or a major anomaly of a single organ, inborn errors of metabolism and congenital viral infections. The present study was registered as a clinical study in the Chinese Clinical Trial Registry (ChiCTR-ORC-16008872) and the Institutional Review Board of Zhongnan Hospital of Wuhan University approved the study (approval no. 2015019), and all guardians signed informed consents.

Intervention protocol in hyperbilirubinemia. The intervention was conducted according to the time of neonatal jaundice onset, the causes of hyperbilirubinemia and the serum bilirubin levels. Neonates with hyperbilirubinemia were divided into four groups: conventional intensive single-side phototherapy (halogen lamps) alone (used in all jaundice neonates); phototherapy combined with IVALB (used for unidentified pathological hyperbilirubinemia with total serum bilirubin (TSB) $>300 \mu \mathrm{mol} / 1]$ : phototherapy combined with IVIG (used for jaundiced patients with blood group incompatibility with TSB $<300 \mu \mathrm{mol} / 1)$; and phototherapy combined with IVIG+IVALB (for blood group incompatibility with TSB $>300 \mu \mathrm{mol} / \mathrm{l}$ ). The interference protocols were performed as follows: Phototherapy for $12 \mathrm{~h} /$ day, for 3 days; IVIG $1 \mathrm{~g} / \mathrm{kg} /$ day for 2 days; and IVALB $1 \mathrm{~g} / \mathrm{kg}$ (Fig. 1). A total of 31 of 178 neonates with TSB $>300 \mu \mathrm{mol} / 1$ did not receive IVALB or IVIG due to refusal of blood products by parents.

Data were recorded from the clinical database of infants admitted to the NICU. Clinical characteristics were documented at the time of enrollment, including sex, age (in days after birth), gestation age at delivery, delivery manner and birth weight. Blood samples were collected, and concentrations of serum GLB, ALB, TSB and direct serum bilirubin (DSB) were examined twice, at the first day of hospitalization and the fourth day following phototherapy with or without IVIG and/or IVALB treatment. In the phototherapy-alone group, patients received an equal amount of a $5 \%$ glucose intravenous infusion. The human IVIG $(\mathrm{pH} \mathrm{4,5 \% ,50} \mathrm{ml,}$ $\left.4^{\circ} \mathrm{C}\right)$ and IVALB $(20 \%, 50 \mathrm{ml}$, room temperature) products used in the present study were provided by Wuhan Institute of Biological Products Co., Ltd. (Wuhan, China). The IVIG treatment contained $95 \%$ monomeric $\mathrm{IgG}$ and no aggregates when applied according to the manufacturer's protocol.
Table I. Characteristics of neonatal hyperbilirubinemia.
Characteristics

Sex

\section{Male}

Female

Age at enrollment, days

Delivery

Natural labor

Cesarean

Gestational age, weeks

Body weight, g

Serum TSB at enrollment, $\mu \mathrm{mol} / 1$

DSB at enrollment, $\mu \mathrm{mol} / \mathrm{l}$

Pathological cause of

hyperbilirubinemia

Idiopathic

Blood group incompatibility

Infection

Polycythemia

Extravascular bleeding

Therapy

Phototherapy alone

Phototherapy+IVIG

Phototherapy+IVALB

Phototherapy+IVIG+IVALB
Median (range) or $\mathrm{n}(\%)$

$240(55.8)$

$190(44.2)$

$4(1-28)$

$183(42.6)$

$247(57.4)$

$38(37-42)$

3,200 (900-4,800)

$281.3(100.7-536.1)$

$20.6(1.5-87.2)$

$363(84.4)$

50 (11.6)

$11(2.6)$

4 (1.0)

$250(58.1)$

$20(4.7)$

$104(24.2)$

$56(13)$
$2(0.5)$

IVIG, intravenous immunoglobulin; IVABL, intravenous albumin.

Laboratory examination. The serum was separated from blood samples in the dark and centrifuged $(1,509 \times \mathrm{g}$ for $10 \mathrm{~min}$ at room temperature). Serum TSB, DSB, ALB and GLB were measured in all hyperbilirubinemia neonates at $1 \mathrm{~h}$ after admission and the fourth day after the initiation of treatment using a Beckman AU5800 biochemistry analyzer (Beckman Coulter, Inc., Brea, CA, USA).

Statistical analysis. Data are presented as mean \pm standard deviation or number (\%). Pearson correlation analysis was performed between the variances of infant age with ALB and GLB levels. Continuous variables were compared between the groups using the two-tailed Student's t-test and one-way analysis of variance with the Student-Newman-Keuls method. Statistical analysis was performed using GraphPad Prism 5 (GraphPad Software, Inc., La Jolla, CA, USA). P<0.05 was considered to indicate a statistically significant difference.

\section{Results}

Characteristics of neonatal hyperbilirubinemia. A total number of 430 Chinese full-term newborns were enrolled in the present study. Patient clinical characteristics are summarized in Table I. A total of 302 cases were admitted at 1-6 days after birth, 42 cases at 7-10 days, 43 cases at 11-15 days and 43 cases 


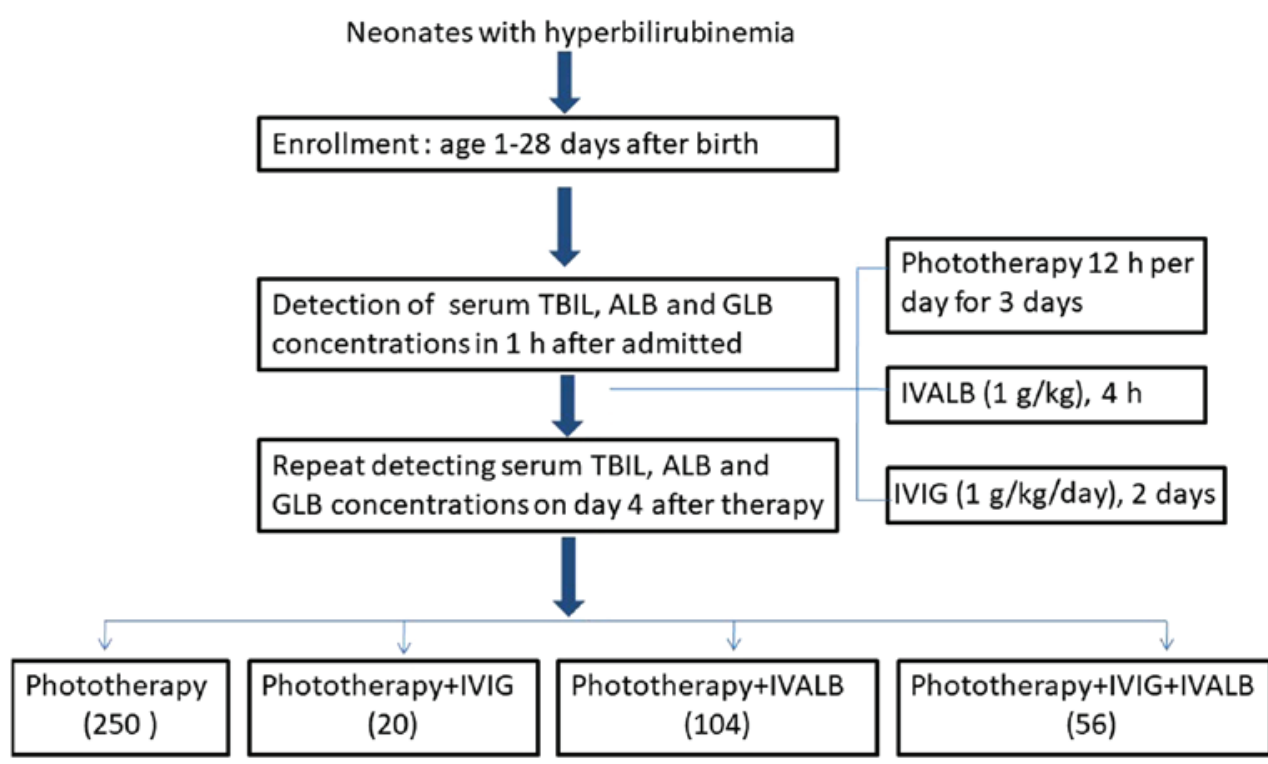

Figure 1. Intervention protocol of neonatal hyperbilirunemia. ALB, albumin; GLB, globulin; VIG, intravenous immunoglobulin; IVABL, intravenous albumin.

at 16-28 days. The primary cause of hyperbilirubinemia was idiopathic $(84.4 \%)$, followed by blood group incompatibility $(11.6 \%)$ and bacterial infection, identified as sepsis $(2.6 \%)$. Median of TSB and DSB levels prior to interference were 281.3 (100.7-536.1) $\mu \mathrm{mol} / \mathrm{l}$ and 20.6 (1.5-87.2) $\mu \mathrm{mol} / 1$, respectively.

Associations between ALB, GLB and TSB levels in neonatal hyperbilirubinemia. Prior to the intervention, the association between neonatal hyperbilirubinemia baseline ALB, GLB and TSB levels was evaluated. Pearson correlation analysis indicated no correlation between ALB and TSB levels ( $r=0.072$; $\mathrm{P}=0.134)$. Conversely, GLB and TSB levels were significantly positively correlated $(r=0.249 ; \mathrm{P}<0.01)$. The linear regression analyses indicated that the ALB concentrations increased along with the age [95\% confidence interval (CI); 0.073, 0.205; $\mathrm{P}<0.001$, and GLB concentration gradually decreased with age from day 1 to day 28 after birth (95\% CI; -0.152, -0.0159; Fig. 2). Following 3 days of treatment, TSB concentrations decreased from $299.6 \pm 83.9$ to $163.6 \pm 57.6 \mu \mathrm{mol} / 1(\mathrm{P}<0.001)$. These data imply that neonatal hyperbilirubinemia incurs a decrease in GLB levels during the perinatal period.

When compared with the effect of different methods of intervention on serum ALB and GLB levels, as indicated in Table IIA, there was no significant difference of the serum ALB concentration in the phototherapy-alone and phototherapy combined with IVIG treatment $(\mathrm{P}>0.05)$, and ALB levels increased in groups who received additional IVALB. By contrast, the GLB levels decreased significantly from $21.3 \pm 4.1$ to $18.5 \pm 4.2 \mathrm{~g} / \mathrm{l}$ in the phototherapy group (Table IIB), and $23.0 \pm 3.9$ to $16.6 \pm 4.5 \mathrm{~g} / 1$ in the group of phototherapy combined with IVALB $(\mathrm{P}<0.001)$. There was no significant difference in GLB levels in groups receiving IVIG. All infants exhibited marked decreases in TSB concentration compared with their baseline levels (Table IIC).

Phototherapy promotes the decrease in GLB levels in neonatal hyperbilirubinemia. To exclude the effect of IVIG and ALB infusion on serum GLB levels, the effect of phototherapy with

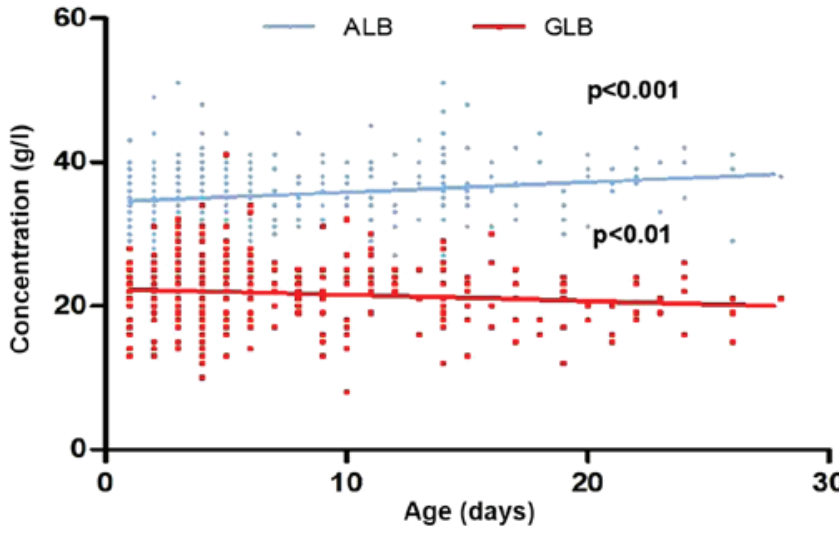

Figure 2. Linear regression analysis of ALB and GLB baseline serum concentrations. ALB, albumin; GLB, globulin.

and without IVALB intervention on serum GLB concentration along with neonatal age was evaluated. As demonstrated in Fig. 3A, 250 patients received phototherapy only, and the ALB levels indicated no change following the intervention $(P>0.05)$. However, the GLB level decreased significantly (Fig. 3B) following the treatment, and the degree of decrease in concentration increased with age $(\mathrm{P}<0.001)$. Among these cases, 4 neonates admitted were identified as having a bacterial infection, with severe sepsis on the fifth day following phototherapy, requiring the administration of antibiotics. The maximum decrease in GLB levels occurred in age range of 16-28 days, which exhibited a $4.7 \mathrm{~g} / 1$ decrease (22\%) in comparison with the first day of admission.

Fig. 3C describes the 104 infants who received phototherapy combined with IVALB at the various birth ages. The ALB levels increased in correspondence with increases in age, while there was no significant change compared with their original levels $(\mathrm{P}>0.05)$. Conversely, the decrease in GLB level was significantly decreased at each age (Fig. 3D; $P<0.001)$. The maximum decrease in GLB levels occurred between the ages of 16-28 days, which exhibited a $4.5 \mathrm{~g} / 1$ decrease compared with 
Table II. ALB, GLB and TSB levels in each treatment group.

A, ALB concentrations in different intervention groups

\begin{tabular}{|c|c|c|c|c|}
\hline Intervention group & $\mathrm{N}$ & ALB-0 (g/l) & ALB-1 (g/l) & P-value \\
\hline Phototherapy alone & 250 & $35.4 \pm 3.9$ & $35.2 \pm 3.6$ & 0.582 \\
\hline Phototherapy+IVIG & 20 & $34.3 \pm 4.3$ & $32.4 \pm 5.3$ & 0.586 \\
\hline Phototherapy+IVALB & 104 & $35.7 \pm 4.8$ & $39.3 \pm 3.6$ & $<0.010$ \\
\hline Phototherapy+IVIG+IVALB & 56 & $34.8 \pm 4.3$ & $36.5 \pm 3.3$ & 0.009 \\
\hline
\end{tabular}

B, GLB concentrations in different intervention groups

\begin{tabular}{lrrrr}
\hline Intervention group & $\mathrm{N}$ & GLB-0 $(\mathrm{g} / \mathrm{l})$ & GLB-1 (g/l) & P-value \\
\hline Phototherapy alone & 250 & $21.3 \pm 4.1$ & $18.5 \pm 4.2$ & $<0.010$ \\
Phototherapy+IVIG & 20 & $22.4 \pm 3.5$ & $23.8 \pm 5.6$ & 0.499 \\
Phototherapy+IVALB & 104 & $23.0 \pm 3.9$ & $16.6 \pm 4.5$ & $<0.010$ \\
Phototherapy+IVIG+IVALB & 56 & $22.9 \pm 4.7$ & $22.6 \pm 4.3$ & 0.451 \\
\hline
\end{tabular}

C, TSB concentrations in different intervention groups

\begin{tabular}{lrcc}
\hline Intervention group & $\mathrm{N}$ & TSB-0 $(\mu \mathrm{mol} / \mathrm{l})$ & TSB-1 $(\mu$ mol/l) \\
\hline Phototherapy alone & 250 & $230.9 \pm 54.0^{\mathrm{a}}$ & $149.4 \pm 60.6^{\mathrm{b}}$ \\
Phototherapy+IVIG & 20 & $222.3 \pm 57.8^{\mathrm{a}}$ & $144.1 \pm 27.3^{\mathrm{b}}$ \\
Phototherapy+IVALB & 104 & $353.1 \pm 44.5$ & $182.0 \pm 34.6^{\mathrm{b}}$ \\
Phototherapy+IVIG+IVALB & 56 & $325.7 \pm 71.8$ & $172.5 \pm 46.2^{\mathrm{b}}$
\end{tabular}

GLB, globulin; ALB, albumin; TSB, total serum bilirubin; IVIG, intravenous immunoglobulin; IVABL, intravenous albumin; GLB-0, serum GLB levels at admission; GLB-1, serum GLB levels following intervention; ALB-0, serum ALB levels at admission; ALB-1, serum ALB levels following intervention; TSB-0, TSB levels at admission; TSB-1, TSB levels following intervention. ${ }^{\mathrm{a}} \mathrm{P}<0.001 \mathrm{vs}$. IVALB and IVIG+IVALB; ${ }^{b} \mathrm{P}<0.001$ vs. TSB. Data are presented as mean \pm standard deviation. Statistical analysis was performed using analysis of variance.

their baseline level. These results indicate that phototherapy may be the primary mechanism for the decreasing neonatal serum GLB levels, particularly in infants aged $>16$ days old, while additional IVALB promoted the decrease, along with increasing birth ages. As a result, the effect of phototherapy on GLB levels was correlated with patient age; older infants (16-28 days old) exhibited a greater decrease in GLB levels compared with younger patients ( $<16$ days old).

\section{Discussion}

The present study summarized the observation that serum GLB levels decrease in response to intensive phototherapy, with or without IVALB infusion in full-term neonates with hyperbilirubinemia. In addition, age may be an important factor in the decrease of GLB levels.

Phototherapy is popular for the treatment of neonatal hyperbilirubinemia, and occasionally in combination with IVIG and IVALB infusion for severe cases $(6,9,16)$. IVIG and/or IVALB are effective for neonates with hemolytic hyperbilirubinemia and for preventing neurodevelopmental abnormalities or kernicterus. The present study adopted phototherapy+IVALB treatment strategy for certain hyperbilirubinemia cases as these infants exhibited higher serum bilirubin levels compared with normal physiological ranges; these increased levels may be attributed to hyperhemoglobinemia, which causes prolonged hyperbilirubinemia and increases the risk of bilirubin encephalopathy. Therefore, the IVALB was used as a preventive treatment. In the present study, although there was different baseline TSB levels between the phototherapy-alone and phototherapy+IVALB groups, there was no difference in their baseline ALB and GLB levels. Phototherapy was conducted for each infant, and IVIG was added if neonates presented with hemolytic hyperbilirubinemia at first day of birth, or IVALB was added for neonates with serum TSB levels $>300 \mu \mathrm{mol} / 1$ at the time of admission, and the combination of IVIG+IVALB was administered in patients with hemolytic hyperbilirubinemia with TSB $>300 \mu \mathrm{mol} / 1$. Using this protocol, no infant mortalities were recorded, and no patients developed kernicterus or required blood transfusion. However, four neonates who had a negative blood culture at their initial admission were identified as having bacterial infection with severe sepsis (Escherichia coli) on the fifth day following phototherapy alone ( 3 cases) or combined with IVALB ( 1 case), requiring the administration 

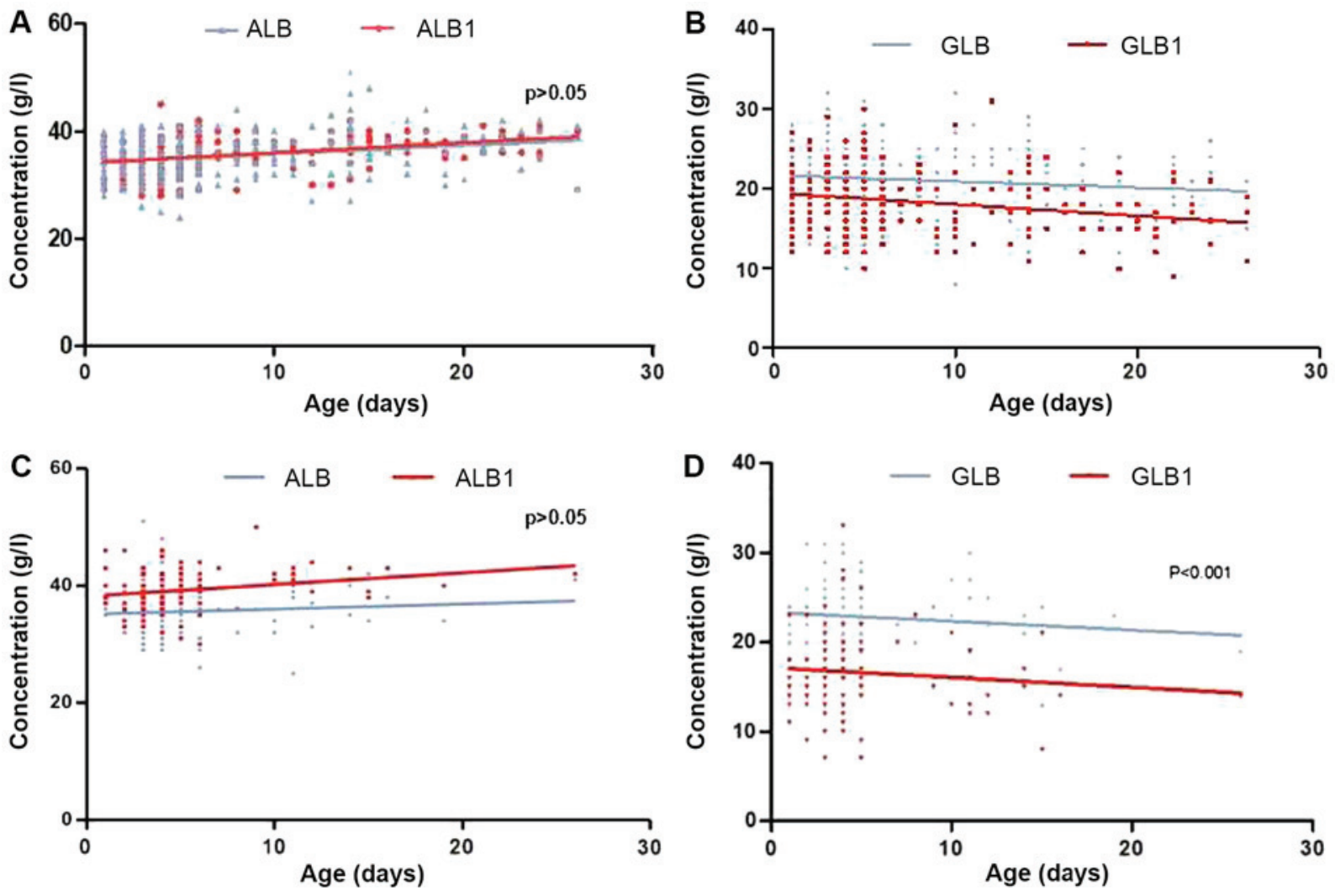

Figure 3. ALB, ALB1, GLB and GLB1 serum concentration measurements at baseline and on the fourth day following treatment. (A) ALB levels prior and subsequent to phototherapy alone. (B) GLB levels prior and subsequent to phototherapy alone. (C) ALB levels prior and subsequent to phototherapy combined with intravenous albumin. (D) GLB levels prior and subsequent to phototherapy combined with intravenous albumin. Data were analyzed with an unpaired t-test. GLB, baseline globulin level; ALB, baseline albumin level; GLB-1, serum GLB levels following intervention; ALB-1, serum ALB levels following intervention.

of antibiotics. No side effects were observed in groups who received IVIG.

The GLB proteins are usually classified into four groups, defined as $\alpha-1,-2, \beta$ and $\gamma$ globulins. The optimal range for GLB is $21-23 \mathrm{~g} / 1$ in neonates, which includes $75 \% \gamma$ globulin $(17,18) . \alpha$ and $\beta$ globulins are synthesized in the liver cells and $\gamma$ globulin is produced by B lymphocytes. Among the serum GLB in neonates, $75 \%$ are $\mathrm{IgG}$, and a small number of $\operatorname{IgM}, \operatorname{IgA}$ and $\operatorname{IgD}$ molecules, which are predominantly active against bacteria, and are responsible for the opsonization of microbes and facilitation of their uptake and elimination by phagocytic cells in the immune system. In the present study, the baseline ALB/GLB ratio was 1.7 , and increased to $2.3 \mathrm{~g}$ in the phototherapy-only group, which implies that phototherapy is the primary reason for decreases in GLB, while IVALB additionally promoted the decrease.

Infants acquire maternal $\mathrm{IgG}$ in utero, and then begin to gradually produce $\mathrm{IgG}$ autonomously up to normal levels by 1 year after birth; IgG concentrations decrease progressively until the third month, and then increase again until the sixth month $(19,20)$. However, phototherapy intervention may accelerate the clearance of $\mathrm{IgG}$, which may confer a potential risk of developing humoral immune disorders $(11,12)$. Although it cannot be confirmed that the cases of sepsis were directly associated with the phototherapy, the risks of severe infection appeared to be unavoidable when the ALB/ALB ratio was disrupted (21). It has been demonstrated that phototherapy may cause an increased incidences of infection in neonates by increasing the total number of peripheral white blood cells, which may complicate any existing infections $(22,23)$. Previous study has demonstrated that phototherapy may have harmful effects on the immune system of neonates with hyperbilirubinemia (14). The potential mechanisms of immunological disturbances subsequent to phototherapy treatment involve affecting the Th2/Th1 switch disorder, ultimately causing allergic diseases during childhood and later in life, which may affect the immune system and lead to autoimmunity disorders $(13,24)$. From these studies, it may be inferred that phototherapy is not only associated with cellular immunity, but that it also attenuates humoral immunity.

As demonstrated in the present study, the decrease in neonate GLB levels was associated with phototherapy; the maximum ratio decreased to $30 \%$ compared with baseline levels, particularly in the group of neonates $>16$ days. The mechanism through which phototherapy causes decreases in GLB remains unclear. Previous studies have indicated that ALB may bind to the FcRn and alter the serum half-life of GLB, while ALB may block the FcRn-IgG interaction and increase $\mathrm{IgG}$ catabolism, as FcRn is responsible for protecting IgG from degradation by trafficking $\operatorname{IgG}(15,25)$. Neonates who received IVIG demonstrated an optimal concentration of GLB following treatment (26). However, this does not mean that the use of IVIG during phototherapy 
should be encouraged; conversely, doctors must carefully consider the use of phototherapy in those patients with hyperbilirubinemia that are not high risk, and ensure that sufficient access to breast feeding is available during phototherapy (27-30).

There are certain limitations in the present study: The IgG levels were not measured, and there was no long-term follow-up strategy to define the subsequent immune status of the children who underwent phototherapy during the neonatal period. The final conclusions of the effects of phototherapy on the levels of serum GLB later in childhood are not available at present, but will be observed in future.

Taken together, the results of the present study demonstrated that phototherapy accelerates serum GLB depletion, which implies infants receiving phototherapy have an increased risk of immunosuppression, particularly in newborns aged $>16$ days. Additional IVALB treatment promoted this decrease, along with increased age.

\section{Acknowledgements}

The present study was published as an abstract at the 26th Annual Conference of the Asian Pacific Association for the Study of the Liver conference proceedings.

\section{Funding}

The present study was supported by the National Natural Scientific Fund of China, awarded to Dr Zhao (grant nos. 81170005 and 81670007).

\section{Availability of data and materials}

The datasets used and analyzed during the current study are available from the corresponding author on reasonable request.

\section{Authors' contributions}

JZ performed the initial analysis and drafted the initial manuscript. CW and MZ performed the statistical analysis. DZ contributed to the conception of the study, interpreted the data analysis and finalized the manuscript as submitted. All authors read and approved the final manuscript.

\section{Ethics approval and consent to participate}

The present study was registered as a clinical study in the Chinese Clinical Trial Registry (ChiCTR-ORC-16008872), and the Institutional Review Board of Zhongnan Hospital of Wuhan University approved the study (approval no. 2015019). All guardians provided informed consent.

\section{Patient consent for publication}

All guardians provided informed consent on behalf of infants for the publication of associated data.

\section{Competing interests}

The authors declare that they have no competing interests.

\section{References}

1. American Academy of Pediatrics Subcommittee on Hyperbilirubinemia: Management of hyperbilirubinemia in the newborn infant 35 or more weeks of gestation. Pediatrics 114: 297-316, 2004.

2. Rennie J, Burman-Roy S and Murphy MS; Guideline Development Group: Neonatal jaundice: summary of NICE guidance. BMJ 340: c2409, 2010.

3. Gamaleldin R, Iskander I, Seoud I, Aboraya H, Aravkin A, Sampson PD and Wennberg RP: Risk factors for neurotoxicity in newborns with severe neonatal hyperbilirubinemia. Pediatrics 128: e925-e931, 2011.

4. Hosono S, Ohno T, Kimoto H, Nagoshi R, Shimizu M and Nozawa M: Effects of albumin infusion therapy on total and unbound bilirubin values in term infants with intensive phototherapy. Pediatr Int 43: 8-11, 2001.

5. Huizing K, Røislien J and Hansen T: Intravenous immune globulin reduces the need for exchange transfusions in Rhesus and AB0 incompatibility. Acta Paediatr 97: 1362-1365, 2008.

6. Hosono S, Ohno T, Kimoto H, Nagoshi R, Shimizu M, Nozawa M and Harada K: Follow-up study of auditory brainstem responses in infants with high unbound bilirubin levels treated with albumin infusion therapy. Pediatr Int 44: 488-492, 2002.

7. Ahlfors CE and Wennberg RP: Bilirubin-albumin binding and neonatal jaundice. Semin Perinatol 28: 334-339, 2004.

8. Yokota T, Morioka I, Kodera T, Morisawa T, Sato I, Kawano S, Koda T, Matsuo K, Fujioka K, Morikawa S, et al: Novel treatment strategy for Japanese newborns with high serum unbound bilirubin. Pediatr Int 55: 54-59, 2013.

9. Xiong T, Chen $\mathrm{H}$ and $\mathrm{Mu} \mathrm{D}$ : Effect of pre-exchange albumin infusion on neonatal hyperbilirubinaemia and long-term developmental outcomes. Cochrane Database of Systematic Reviews, Issue 2, Art. no. CD011001, 2014.

10. Khera S and Gupta R: Incidence of thrombocytopenia following phototherapy in hyperbilirubinemic neonates. Med J Armed Forces India 67: 329-332, 2011.

11. Huang L, Bao Y, Xu Z, Lei X, Chen Y, Zhang Y and Zhang J: Neonatal bilirubin levels and childhood asthma in the US Collaborative Perinatal Project, 1959-1965. Am J Epidemiol 178: 1691-1697, 2013.

12. Sun HL, Lue KH and Ku MS: Neonatal jaundice is a risk factor for childhood allergic rhinitis: A retrospective cohort study. Am J Rhinol Allergy 27: 192-196, 2013.

13. Abourazzak S, Bouharrou A and Hida M: Jaundice and urinary tract infection in neonates: Simple coincidence or real consequence? Arch Pediatr 20: 974-978, 2013 (In French).

14. Chang HY, Cheng KS, Liu YP, Hung HF and Fu HW: Neonatal infected subgaleal hematoma: an unusual complication of early-onset E. coli sepsis. Pediatr Neonatol 56: 126-128, 2013.

15. Andersen JT, Dalhus B, Cameron J, Daba MB, Plumridge A, Evans L, Brennan SO, Gunnarsen KS, Bjørås M, Sleep D, et al: Structure-based mutagenesis reveals the albumin-binding site of the neonatal Fc receptor. Nat Commun 3: 610-610, 2012.

16. Maisels MJ and McDonagh AF: Phototherapy for neonatal jaundice. N Engl J Med 358: 920-928, 2008.

17. Hyvarinen M, Zeltzer P, Oh W and Stiehm ER: Influence of gestational age on serum levels of alpha-1 fetoprotein, IgG globulin, and albumin in newborn infants. J Pediatr 82: 430-437, 1973.

18. Salimonu LS, Ladipo OA, Adeniran SO and Osukoya BO: Serum immunoglobulin levels in normal, premature and postmature newborns and their mothers. Int J Gynaecol Obstet 16: 119-123, 1978-1979.

19. Ballow M, Cates KL, Rowe JC, Goetz C and Desbonnet C: Development of the immune system in very low birth weight (less than $1500 \mathrm{~g}$ ) premature infants: Concentrations of plasma immunoglobulins and patterns of infections. Pediatr Res 20: 899-904, 1986.

20. Drossou V,KanakoudiF,DiamantiE, Tzimouli V,Konstantinidis T, Germenis A, Kremenopoulos G and Katsougiannopoulos V: Concentrations of main serum opsonins in early infancy. Arch Dis Child Fetal Neonatal Ed 72: F172-F175, 1995.

21. Keller MA and Stiehm ER: Passive immunity in prevention and treatment of infectious diseases. Clin Microbiol Rev 13: 602-614, 2000.

22. Jafarzadeh M: mohammadzadeh A. Should urine culture be considered in the hyperbilirubinemia workup of a neonate. JCCM 4: 136-138, 2009. 
23. Shahian M, Rashtian P and Kalani M: Unexplained neonatal jaundice as an early diagnostic sign of urinary tract infection. Int J Infect Dis 16: e487-e490, 2012

24. Apaydin K, Ermis B, Arasli M, Tekin I and Ankarali H: Cytokines in human milk and late-onset breast milk jaundice. Pediatr Int 54: 801-805, 2012.

25. Eyada IK, El Saie AL, Ibrahem GA and Riad NM: Effect of phototherapy on B and T lymphocytes in Egyptian infants suffering from neonatal jaundice. Allergol Immunopathol (Madr) 45: 290-296, 2017.

26. Liu P, Li L, Fan P, Zheng J and Zhao D: High-dose of intravenous immunoglobulin modulates immune tolerance in premature infants. BMC Pediatr 18: 74, 2018.

27. Chang RJ, Chou HC, Chang YH, Chen MH, Chen CY, Hsieh WS and Tsao PN: Weight loss percentage prediction of subsequent neonatal hyperbilirubinemia in exclusively breastfed neonates. Pediatr Neonatol 53: 41-44, 2012.
28. El Sakka A, Imam SS, El Barbary M and Ibrahium W: Does Type of Infant Feeding Affect Phototherapy for Neonatal Hyperbilirubinemia? SAGE J 4: 334-339, 2012.

29. Cuperus FJ, Schreuder AB, van Imhoff DE, Vitek L, Vanikova J, Konickova R, Ahlfors CE, Hulzebos CV and Verkade HJ: Beyond plasma bilirubin: The effects of phototherapy and albumin on brain bilirubin levels in Gunn rats. J Hepatol 58: 134-140, 2013.

30. Cohen RS, Wong RJ and Stevenson DK: Understanding neonatal jaundice: A perspective on causation. Pediatr Neonatol 51: $143-148,2010$. 